\title{
BOUNDARY BEHAVIOR OF A NONPARAMETRIC SURFACE OF PRESCRIBED MEAN CURVATURE NEAR A REENTRANT CORNER
}

\author{
ALAN R. ELCRAT AND KIRK E. LANCASTER
}

\begin{abstract}
Let $\Omega$ be an open set in $\mathbf{R}^{2}$ which is locally convex at each point of its boundary except one, say $(0,0)$. Under certain mild assumptions, the solution of a prescribed mean curvature equation on $\Omega$ behaves as follows: All radial limits of the solution from directions in $\Omega$ exist at $(0,0)$, these limits are not identical, and the limits from a certain half-space $(H)$ are identical. In particular, the restriction of the solution to $\Omega \cap H$ is the solution of an appropriate Dirichlet problem.
\end{abstract}

0. Introduction. We consider here the behavior of a generalized solution of the equation for surfaces of prescribed mean curvature at an inner corner of the boundary where the solution is discontinuous. This work is a generalization of the previous work of the second author $[\mathbf{8}]$, which dealt with the minimal surface equation. It was shown there that all radial limits exist and that they are constant in directions coming from a half-space. Here we find that the same result holds for (nonparametric) surfaces of prescribed mean curvature.

1. Preliminaries. Let $\Omega$ be a bounded, open, connected, simply connected subset of $\mathbf{R}^{2}$ with $N=(0,0) \in \partial \Omega$ such that $\Omega$ is locally convex at each point of $\partial \Omega \backslash\{N\}$. Let $H(x, y, t)$ be a continuous function on $\Omega \times \mathbf{R}$ and $\phi \in C^{0}(\partial \Omega)$. We will make a number of assumptions which will hold throughout this work.

Assumptions. (A) The equation

$$
(p / W)_{x}+(q / W)_{y}=2 H(x, y, z(x, y))
$$

has a solution $z=f \in C^{2}(\Omega)$, where $p=z_{x}, q=z_{y}$, and $W^{2}=1+p^{2}+q^{2}$.

(B) $f \in C^{0}(\bar{\Omega} \backslash\{N\})$ and $f=\phi$ on $\partial \Omega \backslash\{N\}$.

(C) $F \notin C^{0}(\bar{\Omega})$.

(D) There exists $Q>0$ such that $|H(x, y, f(x, y))| \leq Q$ for all $(x, y) \in \Omega$.

(E) The area of the graph of $f$ over $\Omega$ is finite.

A function $f$ satisfying the above conditions can be obtained as a generalized solution of the Dirichlet problem for (1) in $\Omega$ with data $\phi$.

One method for obtaining such solutions is to minimize the functional

$$
\iint_{\Omega}\left(1+f_{x}+f_{y}\right)^{1 / 2} d x d y+\iint_{\Omega} \int_{0}^{f} 2 H(x, y, t) d t d x d y+\int_{\partial \Omega}|f-\phi|
$$

Received by the editors September 2, 1984.

1980 Mathematics Subject Classification (1985 Revision). Primary 35J67, 35J65; Secondary $35 \mathrm{~J} 20$. 
in $\operatorname{BV}(\Omega)$. Suppose that $\Omega$ has a locally Lipschitz boundary, $H$ is Lipschitz and nondecreasing in $t$, and $H_{0}(x, y)=2 H(x, y, 0)$ satisfies

$$
\left|\iint_{A} H_{0}(x, y) d x d y\right| \leq(1-\varepsilon) \iint_{A}\left|D \chi_{A}\right|
$$

for all Caccioppoli sets $A \subset \Omega$, where $0<\varepsilon<1$ is fixed and $\chi_{A}$ is the characteristic function of $A$. Then it is shown in [4] that there is a solution of (1) in $\Omega$ satisfying (E) (see also [5, Theorem 1.1]). Further, if $\partial \Omega \backslash\{N\}$ is smooth and its curvature $\kappa(x, y)$ satisfies

$$
|2 H(x, y, f(x, y))| \leq \kappa(x, y)
$$

at each point, then (B) also holds.

On the other hand, if $H=H(x, y)$ and $H$ has a bounded gradient, then the Perron method can be used as was done in [9] for $H$ constant. (The essential ingredient necessary for the extension of Serrin's work to variable $H$ is an interior gradient estimate and this is available in [7 and 10].) In order to use Perron's method, we need a bounded supersolution so that we know that the upper Perron class is nonempty. (A bounded subsolution would do just as well.) The results in [5] enable us to give sharp conditions on when such a supersolution can be found and also show that the two methods are closely related. If $H=H(x, y)$ is Lipschitz and $\Omega$ has a locally Lipschitz boundary, then Giusti shows that a necessary and sufficient condition for the existence of a solution of (1) in $\Omega$ is

$$
\left|2 \iint_{A} H(x, y) d x d y\right|<\iint\left|D \chi_{A}\right|
$$

for all Caccioppoli sets $A$ strictly contained in $\Omega$ with positive measure. If the strict inequality holds when $A=\Omega$, then it is shown that (2) holds. (In the boundary case $\left|2 \iint_{\Omega} H\right|=\iint_{\Omega}\left|D \chi_{\Omega}\right|=H_{1}(\partial \Omega)$, the unique (up to a constant) solution of $(1)$ is bounded iff $\kappa(x, y)<2 H(x, y)$.) We see then that in many cases the Perron method can be applied if and only if the variational method can.

Finally, if $H=H(x, y)$, using results from $[3, \S 15]$, we can give domains $\Omega$ and boundary data $\phi$ such that $(\mathrm{C})$ must hold. In particular, if $N$ is an inner boundary point of $\Omega[\mathbf{3}]$, then a bound for the value of $z(N), z \in C^{0}(\bar{\Omega})$, satisfying (1) can be given which depends only on values of $z$ at points of $\partial \Omega$ bounded away from $N$.

2. A parametric representation of $z=f(x, y)$. Define

$$
\begin{aligned}
& S=\{(x, y, f(x, y)) \mid(x, y) \in \bar{\Omega} \backslash\{N\}\}, \\
& \Gamma=\{(x, y, \phi(x, y)) \mid(x, y) \in \partial \Omega\},
\end{aligned}
$$

and $S_{0}=S \backslash \Gamma$. Let $T=\{(0,0, z) \mid z$ is real $\}$ be the $z$-axis. We need some parameter domains, so set $E=\left\{(u, v) \mid u^{2}+v^{2}<1\right\}, B=\{(u, v) \in E \mid v>0\}, \partial^{\prime} B=$ $\left\{(u, v) \mid u^{2}+v^{2}=1, v>0\right\}, \partial^{\prime \prime} B=\{(u, 0) \mid-1<u<1\}$, and $B^{\prime}=B \cup \partial^{\prime} B$. Let $P=(0,0, \phi(0,0))$ and $\Gamma_{0}=\Gamma \backslash\{P\}$.

LEMMA 2.1. There is a vector $X \in C^{0}\left(B^{\prime}: R^{3}\right) \cap C^{2}\left(B: R^{3}\right)$ with the following properties:

(i) $X$ is a homeomorphism of $B$ onto $S_{0}$.

(ii) $X$ maps $\partial^{\prime} B$ strictly monotonically onto $\Gamma_{0}$.

(iii) $X$ is conformal on $B$, i.e. $X_{u} \circ X_{v}=0, X_{u}^{2}=X_{v}^{2}$ on $B$. 
(iv) $X_{u u}+X_{v v}=2 H(X) X_{u} \times X_{v}$ on $B$.

(v) If we write $X(u, v)=(x(u, v), y(u, v), z(u, v))$, then $x, y \in C^{0}(\bar{B})$ and $x(u, 0)$ $=y(u, 0)=0$ for $-1 \leq u \leq 1$.

(vi) As $0 \leq \theta \leq \pi$ increases, $K(\cos (\theta), \sin (\theta))$ moves in a clockwise direction about $\partial \Omega$. Here $K(u, v)=(x(u, v), y(u, v))$.

PROOF. At each point $Q$ of $S_{0}$, there is a neighborhood $U$ of $Q$ in $S_{0}$ and a vector $Y \in C^{2}\left(E: \mathbf{R}^{3}\right)$ such that $Y$ is a homeomorphism from $E$ onto $U, Y$ is conformal on $E$, and $\Delta Y=2 H(Y) Y_{u} \times Y_{v}$. Let us pick the vectors $\{Y\}$ so $\left\{Y_{u} \times Y_{v}\right\}$ gives a consistent orientation to $S_{0}$. We may now regard $S_{0}$ as a Riemann surface with local uniformizing parameters $\left\{Y^{-1}\right\}$. By the uniformization theorem for simply connected Riemann surfaces, there is a global uniformizing parameter $\Phi$ mapping $E$ onto $S_{0}$. This means that $\Phi$ is a homeomorphism of $E$ onto $S_{0}$ and that $Y^{-1} \circ \Phi$ is analytic for each local uniformizing parameter $Y^{-1}$ when we regard the domains of $\Phi$ and $Y$ as being subsets of the complex plane. Since $\Phi=Y \circ\left(Y^{-1} \circ \Phi\right)$ on an open subset of $E$, we see that $\Phi$ is conformal on $E$ and that $\Delta \Phi=2 H(\Phi) \Phi_{u} \times \Phi_{v}$.

Set $\Phi(u, v)=(a(u, v), b(u, v), c(u, v))$ and consider the map $G(u, v)=$ $(a(u, v), b(u, v))$. Notice that $\iint_{E} G_{u}^{2}+G_{v}^{2} d u d v \leq 2 \iint_{E}\left|\Phi_{u}\right|^{2} d u d v=2 A(S)<\infty$, where $A(S)$ is the area of $S$. If we apply the proof of Theorem 2.4 of $[\mathbf{1}]$ to $G$, we see that $G \in C^{0}\left(\bar{E}: \mathbf{R}^{2}\right)$. If we define $\sigma$ as the subset of $\partial E$ which $G$ maps onto $\partial \Omega \backslash\{N\}$, then $\Phi \in C^{0}(E \cup \sigma)$, since $f \in C^{0}(\bar{\Omega} \backslash\{N\})$.

Let $w_{0} \in \partial \Omega$ and set $\Omega(\varepsilon)=\left\{w \in \Omega|| w-w_{0} \mid<\varepsilon\right\}$ and $E(\varepsilon)=G^{-1}(\Omega(\varepsilon))$ for all $\varepsilon>0$. Since $G^{-1}(x, y)=\Phi^{-1}(x, y, f(x, y)), G$ is a homeomorphism of $E$ and $\Omega$. For $\varepsilon>0$ small enough, the open sets $\Omega(\varepsilon)$ and $\Omega \backslash \overline{\Omega(\varepsilon)}$ are connected and simply connected and so $E(\varepsilon)$ and $E \backslash \overline{E(\varepsilon)}$ are connected and simply connected. Thus $\phi \neq \partial E \cap \overline{E(\varepsilon)}$ is a connected arc of $\partial E$. Since $\{E(\varepsilon)\}$ is a nested collection, $\overline{E(\varepsilon)}$ converges to a closed, connected arc $\tau\left(w_{0}\right) \subsetneq \partial E$ as $\varepsilon \rightarrow 0$. Notice that $\tau\left(w_{0}\right)=$ $G^{-1}\left(w_{0}\right)$ by construction and so $G$ is weakly monotonic on $\partial E$. In particular $\sigma$ is connected and $\Phi$ maps $\sigma$ weakly monotonically on $\Gamma_{0}$. Now if we use [6], we see that $\Phi$ maps $\sigma$ strictly monotonically onto $\Gamma_{0}$.

If $\partial E \backslash \sigma$ were a single point, we could use the proof of Lemma 2.2 to show that $\Phi \in C^{0}(\bar{E})$ and so $f \in C^{0}(\bar{\Omega})$, in contradiction to (C). To finish, we need only compose $\Phi$ with a suitable conformal map of $B$ onto $E$ and obtain $X$. Q.E.D.

The proof of the next lemma is a (minor) modification of the proof of Lemma 2.2 of $[8]$. Our proof is self-contained for the sake of clarity.

LEMMA 2.2. $X \in C^{0}(\bar{B})$.

Proof. We need prove only that $z \in C^{0}(\bar{B})$. We will prove that $z$ is uniformly continuous on $B$ and so extends to a function in $C^{0}(\bar{B})$.

Let $\varepsilon>0$. Define $g(x, y)$ as the function whose graph is the upper half-sphere of radius $1 / 2 Q$ centered at $(0,0,0)$. Pick $d>0$ so that $g(0,0)-g(2 d, 0)<\varepsilon / 4$. For some $0<\tau<\min (d, \varepsilon / 4)$, the diameter of the shortest arc on $\Gamma \cup T$ joining two points on $\Gamma \cup T$ is less than $\min (d, \varepsilon / 4)$ whenever the distance between the points is less than $\tau[1$, p. 103]. Define $e(\delta)=4 A(S) / \ln (1 / \delta)$ and pick $\delta>0$ so that $2 \pi e(\delta)<\tau^{2}$.

Let $w_{0}=\left(u_{0}, v_{0}\right) \in B$. For $\delta \leq r \leq \sqrt{\delta}$, set $C_{r}=\left\{w \in \bar{B}|| w-w_{0} \mid=r\right\}$. Let $(r, \theta)$ be polar coordinates at $w_{0}$ and let $\xi(r, \theta)=X\left(w_{0}+r(\cos (\theta), \sin (\theta))\right)$. If 
$C_{r} \cap \partial B \neq \varnothing$, define $\alpha(r)$ and $\beta(r)$ by $0 \leq \alpha(r)<\beta(r) \leq 2 \pi$ and $\{(r, \alpha(r)),(r, \beta(r))\}$ $=C_{r} \cap \partial B$; otherwise $\alpha(r)=0$ and $\beta(r)=2 \pi$. Set

$$
p(r)=\int_{\alpha(r)}^{\beta(r)}\left|\xi_{\theta}(r, \theta)\right|^{2} d \theta
$$

then

$$
\int_{\delta}^{\sqrt{\delta}} p(r) / r d r \leq 2 A(S)
$$

For some $\rho \in[\delta, \sqrt{\delta}], \ln (1 / \delta) p(\rho) / 2 \leq 2 A(S)$ and so

$$
p(\rho) \leq 4 A(S) / \ln (1 / \delta)=e(\delta) .
$$

If $C_{\rho}^{*}=X\left(C_{\rho}\right)$ and $L_{\rho}=\int_{\alpha(\rho)}^{\beta(\rho)}\left|\xi_{\theta}(\rho, \theta)\right| d \theta$ is the arclength of $C_{\rho}^{\prime}$, then $L_{\rho}^{2} \leq$ $(\beta(\rho)-\alpha(\rho)) p(\rho) \leq 2 \pi e(\delta)<\tau^{2}$ and so $L_{\rho}<\tau<\min (d, \varepsilon / 4)$. If $C_{\rho} \cap \partial B \neq \varnothing$, then the diameter of the shortest arc on $\Gamma \cup T$ joining the ends of $C_{\rho}^{\prime}$ is at most $\min (d, \varepsilon / 4)$. For any $W \subset \bar{B}$, let $W^{*}=K(W)$.

Define $J_{\rho}$ as the component of $B \backslash C_{\rho}$ which contains $w_{0}$. Set

$$
m=\inf \left\{z(u, v) \mid(u, v) \in C_{\rho} \text { or }(u, v) \in \partial^{\prime} B \cap \bar{J}_{\rho}\right\}
$$

and

$$
M=\sup \left\{z(u, v) \mid(u, v) \in C_{\rho} \text { or }(u, v) \in \partial^{\prime} B \cap \bar{J}_{\rho}\right\} .
$$

Let $U=K\left(J_{\rho}\right)$ and $D_{\rho}=C_{\rho} \cup\left(\bar{J}_{\rho} \cap \partial^{\prime} B\right)$. Notice that $X\left(J_{\rho}\right)$ is the graph of $f$ over $U$ and that $\partial U \backslash D_{\rho}^{*}$ is either empty or contains the single point $(0,0)$. Now let us define $H^{\prime}(x, y)=H(x, y, f(x, y))$ for $(x, y) \in \Omega$ and notice that $\left|H^{\prime}(x, y)\right| \leq Q$ for all $(x, y) \in \Omega$. Notice that the diameter of $U$ is less than $L_{\rho}+\operatorname{diam}\left(D_{\rho}^{*} \backslash C_{\rho}^{*}\right)$, so less than $2 d$. Now we apply Lemma 2.2 of [5] (with " $\Omega=U$ ", " $u=M+g-g(2 d, 0)$ ", " $v=f$ ", and " $\left.\Gamma_{1}=\partial U \backslash\{N\} "\right)$ and see that

$$
f(x, y) \leq M+g(x, y)-g(2 d, 0) \leq M+\varepsilon / 4 \text { for }(x, y) \in U .
$$

Since $z(u, v)=f(x(u, v), y(u, v))$, we get $z(u, v)<M+\varepsilon / 4$ for $(u, v) \in J_{\rho}$. Similarly, $-z(u, v) \leq-m+\varepsilon / 4$ for $(u, v) \in J_{\rho}$. Thus $m-\varepsilon / 4<z(u, v)<M+\varepsilon / 4$ for $(u, v) \in J_{\rho}$.

Now the diameter of $X\left(\partial^{\prime} B \cap \bar{J}_{\rho}\right)$ is less than $\varepsilon / 4$ and $L_{\rho}<\varepsilon / 4$, so $M-m<\varepsilon / 2$. If $(u, v) \in B$ and $\left|(u, v)-\left(u_{0}, v_{0}\right)\right|<\delta$, then $(u, v) \in J_{\rho}$ and so $\left|z(u, v)-z\left(u_{0}, v_{0}\right)\right|<$ $M-m+\varepsilon / 2<\varepsilon$. Since $\delta$ is independent of $w_{0}, z$ is uniformly continuous on $B$ and so can be extended to $\bar{B}$ as a continuous function. Q.E.D.

3. Boundary behavior. By $[6], X \in C^{1}\left(B \cup \partial^{\prime \prime} B\right)$ and the branch points of $X$ on $\partial^{\prime \prime} B$ are isolated. We see that

$$
X_{u}(u, 0)=\left(0,0, z_{u}(u, 0)\right) \text { and } X_{v}(u, 0)=\left(x_{v}(u, 0), y_{v}(u, 0), 0\right) .
$$

Let $(r, \theta)$ be polar coordinates centered at $(0,0)$. Then $\Omega=\{(r, \theta) \mid \alpha<\theta<\beta$, $0<r<r(\theta)\}$ for some $-\pi \leq \alpha<0<\beta \leq \pi$ (where we may need to rotate $\Omega$ about $(0,0))$. For each $\alpha<\theta<\beta$, set $x(t)=x(t, \theta)=t \cdot \cos (\theta)$ and $y(t)=y(t, \theta)=$ $t \cdot \sin (\theta)$. We denote the radial limit of $f$ at $(0,0)$ from the direction $\theta$ (if it exists) by

$$
R f(\theta)=\lim _{t \rightarrow 0+} f(x(t, \theta), y(t, \theta)) .
$$


Set $\lambda(t)=(x(t), y(t), f(x(t)), y(t))$ and $\omega(t)=X^{-1}(\lambda(t))$. Notice that $\lambda(t) \rightarrow$ $(0,0, R f(\theta))$ as $t \rightarrow 0+$ if $\lambda(t)$ converges to a point as $t \rightarrow 0+$. Finally define $R f(\alpha)=R f(\beta)=\phi(0,0)$ and $u(\alpha)=-1, u(\beta)=1$.

THEOREM 3.1. For all $\alpha<\theta<\beta$, there is a unique $u(\theta) \in[-1,1]$ such that $\omega(t) \rightarrow(u(\theta), 0)$ as $t \rightarrow 0+$. Also, $u \in C^{0}([\alpha, \beta])$. Thus $R f(\theta)=z(u(\theta), 0)$ exists for all $\alpha \leq \theta \leq \beta$ and $R f \in C^{0}([\alpha, \beta])$.

The proof is the same as the proof of Lemma 3.1 of [8]. The only facts we use in this proof are that $X \in C^{1}\left(B \cup \partial^{\prime \prime} B\right), K$ is a homeomorphism of $B$ and $\Omega$, and the regular points of $X$ on $\partial^{\prime \prime} B$ are dense in $\partial^{\prime \prime} B$. The fact that $X$ maps $\partial^{\prime} B$ (weakly or strongly) monotonically onto $\Gamma_{0}$ is not important and can be replaced by the following fact:

$$
\left(x_{v}(u(\theta), 0), y_{v}(u(\theta), 0)\right)=\left|z_{u}(u(\theta), 0)\right|(\cos (\theta), \sin (\theta))
$$

for $\alpha<\theta<\beta$ and so $u(\theta)$ is weakly monotonic on $(\alpha, \beta)$.

THEOREM 3.2. There exist $\lambda \in[\alpha, \beta-\pi]$ and $u_{0} \in[-1,1]$ such that $u(\theta)=u_{0}$ for all $\theta \in[\lambda, \lambda+\pi]$; also $X$ is strictly monotonic on $\left[-1, u_{0}\right]$ and on $\left[u_{0}, 1\right]$.

PROOF. Suppose $\theta_{0} \in[\beta-\pi, \alpha+\pi]$ and $X(u, 0)$ is not weakly monotonic on $-1 \leq u \leq u\left(\theta_{0}\right)$. Then $X$ has a branch point at $(b, 0)$ for some $-1<b<u\left(\theta_{0}\right)$ and $z_{u}(u, 0)$ changes sign at $u=b$, say $z_{u}(u, 0)>0$ on $(b-\varepsilon, b)$ and $z_{u}(u, 0)<0$ on $(b, b+\varepsilon)$ for some $\varepsilon>0$. Pick $a$ and $c$ so that $b-\varepsilon<a<b<c<b+\varepsilon$ and $z(a, 0)=z(c, 0)$. Then $a=u(\theta(a)), c=u(\theta(c))$, and $\alpha<\theta(a)<\theta(c)<\alpha+\pi$. Let $\omega$ be a smooth Jordan arc in $B$ from $(a, 0)$ to $(c, 0)$ such that $\sigma=X(\omega)$ is a simple, closed Jordan curve, $\tau=K(\omega)$ is a convex Jordan curve which is smooth except at $(0,0)$, and the curvature $\kappa(x, y)$ satisfies $\kappa(x, y) \geq 2 Q$ for all $(0,0) \neq(x, y) \in \tau$. Let $U$ be the open region bounded by $\tau$. We will use the function $H^{\prime}(x, y)=H(x, y, f(x, y))$ mentioned earlier.

First, let $h \in C^{0}(\bar{U} \backslash\{N\})$ be the unique variational solution of $(p / W)_{x}+$ $(q / W)_{y}=H^{\prime}(x, y)$ in $U$ with $h=f$ on $\tau \backslash\{N\}[4]$. (We know that (2) is satisfied because of $[\mathbf{5}]$.) If we use a barrier argument of Serrin [9, pp. 375-376] together with Lemma 2.2 of [5], we see that $h \in C^{0}(\bar{U})$.

Second, by Lemma 2.2 of [5], we see that $f=h$ on $U$. This implies that $R f(\theta)=h(0,0)$ for all $\theta \in(\theta(a), \theta(c))$, a contradiction. Thus $X(u, 0)$ is weakly monotonic on $-1 \leq u \leq u\left(\theta_{0}\right)$. Using $[6]$, we see that $X(u, 0)$ is strictly monotonic on $-1 \leq u \leq u\left(\theta_{0}\right)$. Similarly, $X(u, 0)$ is strictly monotonic on $u\left(\theta_{0}\right) \leq u \leq 1$. If $\theta_{1}$ is another element of $[\beta-\pi, \alpha+\pi]$, the same argument proves that $X(u, 0)$ is strictly monotonic on $-1 \leq u \leq u\left(\theta_{1}\right)$ and on $u\left(\theta_{1}\right) \leq u \leq 1$. Since $X(-1,0)=$ $X(1,0)=P$, we see that $u\left(\theta_{0}\right)=u\left(\theta_{1}\right)$. Thus $u(\theta)$ is constant on $[\beta-\pi, \alpha+\pi]$.

Suppose now that $\theta_{2}, \theta_{3} \in[\alpha, \beta]$ with $0<\theta_{3}-\theta_{2}<\pi$. The argument above shows that $X$ is strictly monotonic on $\left[u\left(\theta_{2}\right), u\left(\theta_{3}\right)\right]$. Q.E.D.

Let $\theta_{L}=\inf \left\{\theta \in[\alpha, \beta] \mid u(\theta)=u_{0}\right\}$ and $\theta_{R}=\sup \left\{\theta \in[\alpha, \beta] \mid u(\theta)=u_{0}\right\}$. We know that $\theta_{R}-\theta_{L} \geq \pi$. Also $u(\cdot)$ is a homeomorphism of $\left[\alpha^{\prime}, \theta_{L}\right]$ onto $\left[-1, u_{0}\right]$ and of $\left[\theta_{R}, \beta^{\prime}\right]$ onto $\left[u_{0}, 1\right]$, for some $\alpha \leq \alpha^{\prime} \leq \theta_{L}$ and $\theta_{R}<\beta^{\prime} \leq \beta$. Thus

THEOREM 3.3. The radial limits $R f \in C^{0}([\alpha, \beta])$ behave as follows:

(i) The extreme values of $R f(\theta)$ are $R f(\alpha)=R f(\beta)=\phi(0,0)$ and $R f(0)=$ $z\left(u_{0}, 0\right)$. 
(ii) $R f(\theta)$ is moniotonic on $\left[\alpha, \theta_{L}\right]$.

(iii) $R f(\theta)=z\left(u_{0}, 0\right)$ for all $\theta \in\left[\theta_{L}, \theta_{R}\right]$.

(iv) $R f(\theta)$ is monotonic on $\left[\theta_{R}, \beta\right]$.

Let $U$ be open in $\mathbf{R}^{2}$ with $(0,0) \in \partial U, H(x, y, t)$ be continuous on $U \times \mathbf{R}$, $\phi \in C^{0}(\partial U)$, and $f$ be a $C^{2}$ solution of (1) in $U$. Suppose that for some $\varepsilon>0$, the set $\Omega=\left\{(x, y) \in U \mid x^{2}+y^{2}<\varepsilon^{2}\right\}$ is locally convex at every point of $\partial \Omega$ except $(0,0)$ and $f$ satisfies assumptions (B), (D), (E) in $\Omega$. Then $f$ behaves at $(0,0)$ as indicated in Theorem 3.3. As in [8], we make the following

CONJECTURE. If $\theta_{R}-\theta_{L}>\pi$, then $f \in C^{0}(\bar{\Omega})$.

ADDED IN PROOF. The conjecture mentioned above has been proven in Nonparametric minimal surfaces in $\mathbf{R}^{3}$ whose boundaries have a jump discontinuity (preprint) by the second author.

\section{REFERENCES}

1. R. Courant, Dirichlet's principle, conformal mapping, and minimal surfaces, Interscience, New York, 1950.

2. R. Courant and D. Hilbert, Methods of mathematical physics, Vol. 2, Interscience, New York, 1962.

3. R. Finn, Remarks relevant to minimal surfaces, and to surfaces of prescribed mean curvature, J. Analyse Math. 14 (1965), 139-160.

4. C. Gerhardt, Existence, regularity, and boundary behavior of generalized surfaces of prescribed mean curvature, Math. Z. 139 (1974), 173-198.

5. E. Giusti, On the equation of surfaces of prescribed mean curvature, Invent. Math. 46 (1978), 111-137.

6. E. Heinz, Über das Randverhalten quasilinearer elliptischer Systeme mit isothermen Parametern, Math. Z. 113 (1970), 99-105.

7. O. Ladyzhenskaya and N. Ural'tseva, Local estimates for gradients of solutions of nonuniformly elliptic and parabolic equations, Comm. Pure Appl. Math. 23 (1969), 677-703.

8. K. Lancaster, Boundary behavior of a non-parametric minimal surface in $\mathbf{R}^{3}$ at a nonconvex point, Analysis 5 (1985), 61-69.

9. J. Serrin, The Dirichlet problem for surfaces of constant mean curvature, Proc. London Math. Soc. (3) 21 (1970), 361-384.

10. N. Trudinger, Gradient estimates and mean curvature, Math. Z. 131 (1973), 165-175.

Department of Mathematics and Statistics, Wichita State University, WiCHITA, KANSAS 67208 